\title{
A Novel WSNs Localization Algorithm Based on Artificial Fish Swarm Algorithm
}

\author{
http://dx.doi.org/10.3991/ijoe.v12i01.5180 \\ Xiaoying Yang, Wanli Zhang, Qixiang Song \\ Suzhou University, Suzhou, Anhui, China
}

\begin{abstract}
In node localization algorithm in Wireless Sensor Networks (WSNs), the least square method is affected by the measurement error, which leads to position error of the unknown node. In order to solve the problem that the error is too high, we propose a novel WSNs localization algorithm based on artificial fish swarm (AFSA). In the proposed algorithm, artificial fish swarm, which has some advantages such as low requirements for the initial value and parameter setting is not high, the optimization speed is quick and so on, is introduced in position process. Firstly, the distances between nodes are obtained by using the TDOA algorithm. Then the geometrical position of the unknown node is estimated by the artificial fish swarm optimization algorithm. The simulation results show that the algorithm proposed in the paper can reduce the computation amount, get the optimal solution quickly and improve the accuracy of the node without increasing the cost and power consumption. Moreover, the number of beacon nodes is relatively small, so the network cost is reduced to a certain extent.
\end{abstract}

Index Terms-wireless sensor networks, artificial fish swarm algorithm, least square method, node localization

\section{INTRODUCTION}

Wireless Sensor Networks (WSNs) can be applied to many areas, such as medical care, environmental monitoring, military affairs and other fields (Sun et al.,2005, Niculescu et al.,2003, Arivubrakan et al.,2012, Ji et al.,2015, Arivubrakan et al.,2013). The positioning technology has become the support technology of WSNs (Hu et al., 2013). The current positioning algorithms are divided into two categories: range-based and range-free (Zhou et al., 2008). The range-based algorithms have high requirements of hardware of the sensor nodes and the positioning accuracy is high. RSSI, AOA, TOA, and TDOA are typical range-based algorithms. The range-free algorithms have low requirements of hardware of the sensor nodes, but the positioning accuracy is low. DV-Hop algorithm, centroid algorithm, APIT algorithm, etc. are the typical range-free algorithms (Nirupama et al., 2000, Savvides. 2001, Niculescu et al., 2003).

Because of the high accuracy, the range-based algorithms are usually used in the situation which requires higher accuracy. In traditional range-based node localization, node position is obtained by using least squares method to solute nonlinear equation group. Although the method has the characteristics of good estimates, but the influence of measurement error is relatively large. In order to solve the problem that the error is too high, this paper proposes a new WSN algorithm based on artificial fish swarm. By using artificial fish swarm algorithm to solve nonlinear equations, the algorithm can quickly obtain the optimal solution of position of the unknown node, thus improving the positioning accuracy. As the number of beacon nodes is relatively small, so the network cost can be reduced to a certain extent.

\section{BASIC PRINCIPLES OF POSITIONING AlgRITHOM}

Maximum likelihood estimation is a method that uses multiple anchor nodes to locate, also known as the multilateral measurement method (Pi, 2013). As shown in Figure 1 , nodes $1,2,3, \ldots, \mathrm{n}$ are the anchor nodes, their respective coordinates are $\left(x_{1}, y_{1}\right),\left(x_{2}, y_{2}\right) \ldots,\left(x_{n}, y_{n}\right)$ and the distance between the unknown node is $d_{1}, d_{2}, \ldots, d_{n}$ , respectively. Let $(x, y)$ be the coordinate of the unknown node.

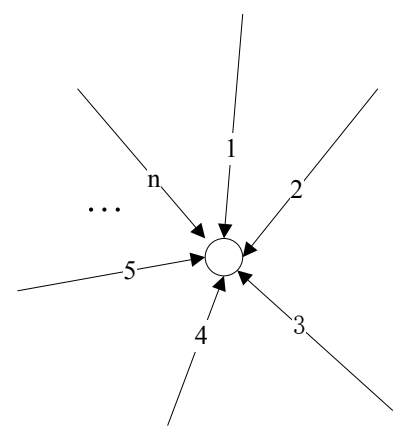

Figure 1. Maximum likelihood estimation

So, there is an equation which is shown as Eq.1.

$$
\left\{\begin{array}{l}
\left(x_{1}-x\right)^{2}+\left(y_{1}-y\right)^{2}=d_{1}^{2} \\
\left(x_{2}-x\right)^{2}+\left(y_{2}-y\right)^{2}=d_{2}^{2} \\
\cdots \\
\left(x_{n}-x\right)^{2}+\left(y_{n}-y\right)^{2}=d_{n}^{2}
\end{array}\right.
$$

From the first equation to the (N-1)-th equation, all of which minus the $\mathrm{N}$-th equation and new equations can be obtained as shown in Eq. 2.

$$
\left\{\begin{array}{l}
x_{1}{ }^{2}-x_{n}{ }^{2}+y_{1}{ }^{2}-2\left(x_{1}-x_{n}\right) x-2\left(y_{1}-y_{n}\right) y=d_{1}{ }^{2}-d_{n}{ }^{2} \\
x_{2}{ }^{2}-x_{n}{ }^{2}+y_{2}{ }^{2}-2\left(x_{2}-x_{n}\right) x-2\left(y_{2}-y_{n}\right) y=d_{2}{ }^{2}-d_{n}{ }^{2} \\
\cdots \\
x_{n-1}{ }^{2}-x_{n}{ }^{2}+y_{n-1}{ }^{2}-2\left(x_{n-1}-x_{n}\right) x-2\left(y_{n-1}-y_{n}\right) y=d_{n-1}{ }^{2}-d_{n}{ }^{2}
\end{array}\right.
$$


Eq.2 can be expressed by linear equations $A X=B$, in

$$
\begin{aligned}
& \text { which } A=\left[\begin{array}{cc}
2\left(x_{1}-x_{n}\right) & 2\left(y_{1}-y_{n}\right) \\
2\left(x_{2}-x_{n}\right) & 2\left(y_{1}-y_{n}\right) \\
\vdots \\
2\left(x_{n-1}-x_{n}\right) 2\left(y_{n-1}-y_{n}\right)
\end{array}\right] \text {, } \\
& B=\left[\begin{array}{l}
x_{1}{ }^{2}-x_{n}{ }^{2}+y_{1}{ }^{2}-y_{n}{ }^{2}+d_{n}{ }^{2}-d_{1}{ }^{2} \\
x_{2}{ }^{2}-x_{n}{ }^{2}+y_{2}{ }^{2}-y_{n}{ }^{2}+d_{n}{ }^{2}-d_{2}{ }^{2} \\
\vdots \\
x_{n-1}{ }^{2}-x_{n}{ }^{2}+y_{n-1}{ }^{2}-y_{n}{ }^{2}+d_{n}{ }^{2}-d_{n-1}{ }^{2}
\end{array}\right], X=\left[\begin{array}{l}
x \\
y
\end{array}\right]
\end{aligned}
$$

Finally, the coordinates of unknown nodes are computed by using least squares method, as shown in Eq.3.

$$
\hat{X}=\left(A^{T} A\right)^{-1} A^{T} B
$$

From the above analysis, it is known that in the calculation of unknown node coordinates, the estimation of the value will inevitably has a large error because of the environment, communication and other factors. The least square method is affected by the measurement error when solving nonlinear equations and it is sensitive to the initial value, which affects the position accuracy of the algorithm.

\section{Node Localization BASED ON ARTificial Fish SWARM}

\section{A. Artificial fish swarm algorithm}

Artificial fish swarm algorithm is an optimization method based on the animal autonomous body which is proposed by $\mathrm{Li}$ in 2003 (Li, 2003). The algorithm simulates behavior of fish feeding, clusters and rear et al. In artificial fish swarm algorithm, the area where fishes live with most numbers is the area where the most abundant nutrient substances are in the water (Xu and Bai, 2012). The algorithm has the advantage of low requirements in the initial value and parameter setting, besides, the optimization speed is quick. Specific behaviors are as follows:

Step 1: Random behavior: artificial fish random moves in the field, when it discovers food, it will gradually fast move in direction of food increasing.

Step 2: Foraging behavior: a behavior that fish swims in direction of much food.

Step 3: Clustering behavior: Fish naturally gather in swarms in the course of swimming to ensure their own survive and avoid harm.

Step 4: Following behavior: a behavior that fish moves to the direction in its visual area.

Step 5: Bulletin board: where record the individual state of the optimal artificial fish.

\section{B. Positioning process of the improved algorithm}

Known from section II, when calculates the coordinates of unknown nodes, the estimated distance $d_{i}$ will inevitably have a large error caused by the environment, communication and other factors, which affect the overall position accuracy of the algorithm. Let the actual distance between the unknown node and the anchor node be $r_{i}$ and the error be $\varepsilon_{i}$, then $\left|r_{i}-d_{i}\right| \leq \varepsilon_{i}$. The smaller the $\varepsilon_{i}$ is, the higher the accuracy of the positioning is. When $\varepsilon_{i} \rightarrow 0$, locating algorithm obtains the optimal solution. Namely, the estimated position is transformed into the problem of finding the minimum value. Take the objective function as:

$$
G=\min \left(\sum_{i=1}^{k}\left(r_{i}-d_{i}\right)\right.
$$

The constraint conditions are:

$$
\left\{\begin{array}{l}
\left|r_{1}-d_{1}\right| \leq \varepsilon_{1} \\
\left|r_{2}-d_{2}\right| \leq \varepsilon_{2} \\
\cdots \\
\left|r_{k}-d_{k}\right| \leq \varepsilon_{k} \\
\sqrt{\left(x-x_{1}\right)^{2}+\left(y-y_{1}\right)^{2}}=d_{1} \\
\sqrt{\left(x-x_{2}\right)^{2}+\left(y-y_{2}\right)^{2}}=d_{2} \\
\cdots \\
\sqrt{\left(x-x_{k}\right)^{2}+\left(y-y_{k}\right)^{2}}=d_{k} \\
r_{k} \geq 0, d_{k} \geq 0, \varepsilon_{k} \geq 0
\end{array}\right.
$$

Artificial fish swarm algorithm is a new bionics algorithm that has characteristics of the low requirement on the initial value, fast convergence speed and quickly obtain the optimal solution. It has been widely applied in many fields. In this paper, we use artificial fish swarm algorithm instead of the least square method to obtain the node location of WSN network. Specific steps are described as follows.

The fitness function of the artificial fish swarm algorithm is defined to measure the pros and cons of the current solution. Fitness function is shown in Eq.6.

$$
f i t=\frac{1}{\alpha+\beta G}
$$

Among them, $\alpha$ and $\beta$ are the constant coefficient of the fitness function, let $\alpha=\beta=1$.

Step 1: Fish swarm initialization. Initial artificial fish, including scale of fish $N$, the visual domain of artificial fish visual, the maximum step size step, crowding factor $\delta$, the number of try times trynumber, and the maximum number of iterations Maxitera.

Step 2: The position of the artificial fish is randomly initialized in the solution range $[0, R]$.

Step3: Foraging behavior: Vector $X_{i}$ expresses the current state of the $i-t h$ artificial fish. The artificial fish can swim freely in the field of vision to jump to a new state $X_{j}$, the formula is:

$$
X_{j}=X_{i}+\operatorname{rand}() \cdot \text { visual }
$$

Where $\operatorname{rand}()$ is a random function which produces $0 \sim 1$. 
If $G_{j}<G_{i}$, indicates that the food concentration of the state $X_{j}$ is better than that of $X_{i}$, then moves a step forward $X_{j}$. At this point, the next state $X_{\text {inext }}$ of $i-t h$ artificial fish is calculated by formula (8).

$$
X_{\text {inext }}=X_{i}+\operatorname{rand}() \cdot \operatorname{step} \cdot \frac{X_{j}-X_{i}}{\left\|X_{j}-X_{i}\right\|}
$$

Otherwise, then randomly select the state $X_{i}$ to determine whether to meet the forward conditions. Such repeated attempts trynumber times, if still not meet the forward conditions. Then randomly move a step.

Step 4: Clustering behavior. Set the current state of artificial fish as $X_{i}$, find the number of partners $N_{f}$ in the current neighborhood $\left(d_{i j}>\right.$ Visual $)$, neighborhood center $X_{c}$. If $\frac{G_{c}}{G_{i} N_{f}}<\delta$, the food concentration near $X_{c}$ is big and fish gathered is small, then move forward a step to the direction of the center of the partner. The status of the artificial fish at the next moment is shown in the formula (9). Otherwise, the artificial fish carry out foraging behavior at the next time.

$$
X_{\text {inext }}=X_{i}+\operatorname{rand}() \cdot \operatorname{step} \cdot \frac{X_{c}-X_{i}}{\left\|X_{c}-X_{i}\right\|}
$$

Step 5: The following behavior. Set current state of artificial fish as $X_{i}$, find the number of partners $N_{f}$ in the current neighborhood (i.e. $d_{i j}<$ visual) and $X_{\max }$ is the largest partner in partners $G_{j}$. If $\frac{G_{\max }}{G_{i} N_{f}}<\delta, X_{\max }$ is with higher food concentration and less crowded around, then move forward a step to the direction of $X_{\max }$. The status of the artificial fish at the next moment is shown in the formula (10). Otherwise, the artificial fish carries out foraging behavior.

$$
X_{\text {inext }}=X_{i}+\operatorname{rand}() \cdot \text { step } \cdot \frac{X_{\text {max }}-X_{i}}{\left\|X_{\text {max }}-X_{i}\right\|}
$$

Step 6: Individuals update themselves through foraging, cluster, following behavior and generate new fish.

Step 7: Use evaluation (6) to evaluate all individuals. If an individual is better than individual on bulletin board, the board is updated to the individual.

Step 8: When optimal solution on the bulletin board reaches satisfactory error bounds, the algorithm ends and output the optimal solution to the variable hopesize, Otherwise turn to step (3), until the maximum number of iterations is reached.

Step 9: Output the current optimal solution, namely, the position estimation of the minimum value of the forecast error.

Step 10: The algorithm ends.

The calculation process is shown in Figure 2.

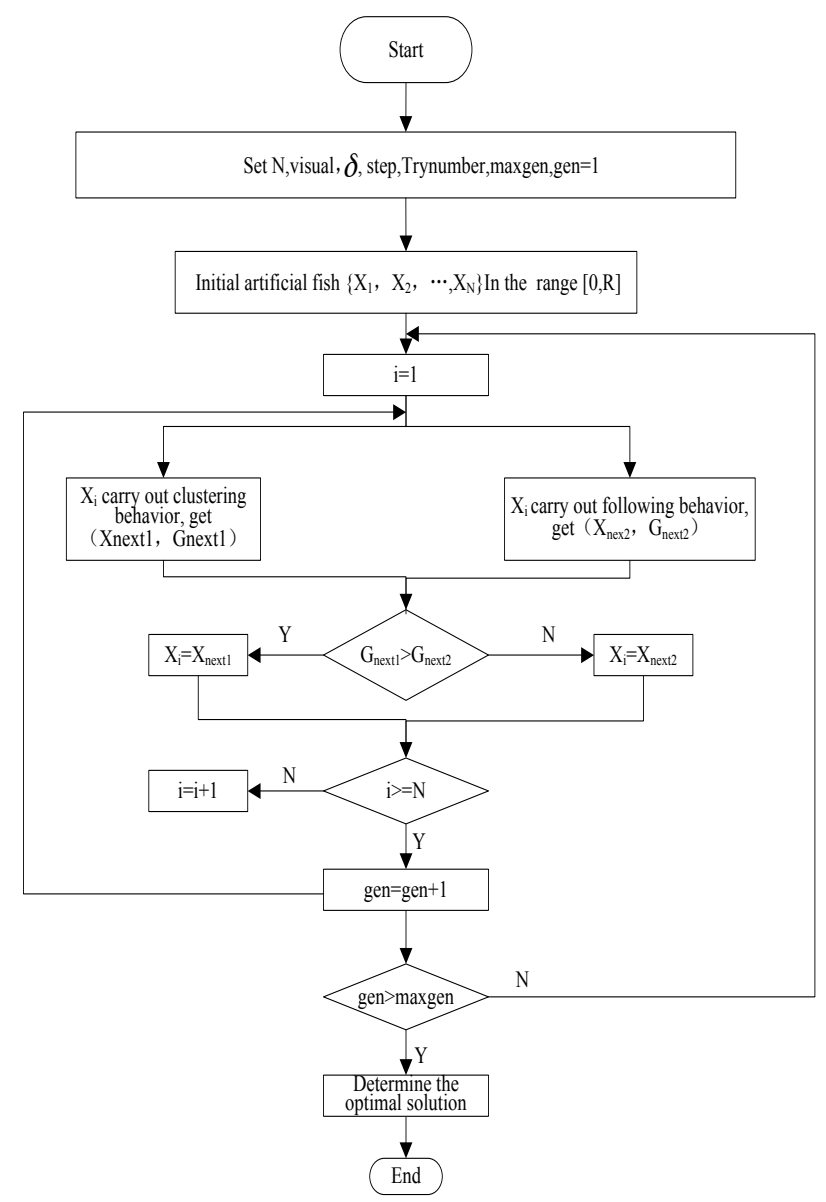

Figure 2. calculation process

\section{Simulation REsults}

\section{A. Simulation environment}

In this section, simulation results are presented and analyzed. In this paper, our performance evaluation focuses on position accuracy of the localization algorithm. In order to do this, we simulated DV-Hop and our proposed algorithm by using MATLAB7.0. Set scale of fish $N$ $=50$, visual field of artificial fish $v i$ sual $=1$, maximum step size step $=0.1$, congestion factor $\delta=0.6$, the number of try times trynumber $=100$, and maximum iteration number Maxitera $=50$. The 100 nodes are randomly distributed in a square area of $100 \times 100$. The communication radius of nodes $R$ is set to $30 \mathrm{~m}$. This paper uses the average positioning error to compare the performance of the two algorithms, which is defined as the formula (11).

$$
\text { Aerr }=\frac{\sum\left(d_{i} / R\right)}{N}
$$

Where $d_{i}$ is the distance between the calculated coordinate and the actual coordinate for the first common node $i$, that is, the node's position error, $d_{i}=\sqrt{\left(x_{t}-x_{e}\right)^{2}+\left(y_{t}-y_{e}\right)^{2}} \cdot\left(x_{t}, y_{t}\right),\left(x_{e}, y_{e}\right)$, respectively, is the true location and evaluated location of the node, $R$ is the communication radius of the node, $N$ is the number of unknown nodes. 


\section{B. Result analysis}

Relationship between anchor node proportion and location coverage: The total number of nodes and the communication radius remain unchanged. The proportion of anchor nodes is increased from $5 \%$ to $30 \%$. Compare the location coverage of AFSA algorithm and that of the least square method. The comparison results are shown in Figure 3. As can be seen from Figure 3, location coverage obtained by AFSA is higher than that by using the least square method. The positioning error of the AFSA algorithm is smaller, so location coverage of it is relatively flat. While the positioning error of using the least square method is larger, so location coverage appears the phenomenon of large and small.

The relationship between the average position error and the ratio of beacon nodes: The total number of nodes remains unchanged. The proportion of anchor nodes is increased from $5 \%$ to $30 \%$. Compare the average position error of AFSA algorithm and that of the least square method. The comparison results are shown in Figure 4. It can be seen from the graph that when the ratio of beacon nodes is low, the positioning errors of the two algorithms are relatively large. With increasing of the proportion of the beacon nodes, the positioning errors of the two algorithms are all decreased. When the number of beacon nodes is same, the average position error of AFSA algorithm is less than that of the least square method.

The relationship between the average position error and range error rate: Ranging error is the difference between the estimated distance and the actual distance between the nodes, and range error rate is the ratio of the range error and node communication radius. The total number of nodes and the communication radius remain unchanged. Compare the average position error of AFSA algorithm and that of the least square methodwith the range error rate changing. The comparison results are shown in Figure 5. It can be seen from Figure 5 that using artificial fish swarm optimization algorithm can achieve a smaller position error than that of the least square method. When the range error rate is increasing, the average position error of node position of the proposed algorithm is smaller than that of the least square method.

The relationship between the average position error and the number of nodes: In the case of different nodes, the average position errors of the artificial fish swarm optimization algorithm and the least square method are compared. The compared results are shown in Figure 6. It can be seen from the graph that when the number of nodes is relatively small, the number of anchor node is relatively small, and the average location error of the AFSA algorithm is smaller than that of the least square method, but when the number of node is larger, the number of anchor node is larger, at this time, the average positioning errors of the two algorithms are reduced and the advantages of the algorithm in this paper are not particularly obvious. But because the artificial fish swarm algorithm has better convergence than that of the least square method, the average positioning errors are relatively small compared with the least square method.

Comparison of energy utilization efficiency of the algorithm: Comparison of the total energy consumption of the two algorithms is shown in Figure7. From the graph, we can see that energy consumption of the AFSA algorithm is

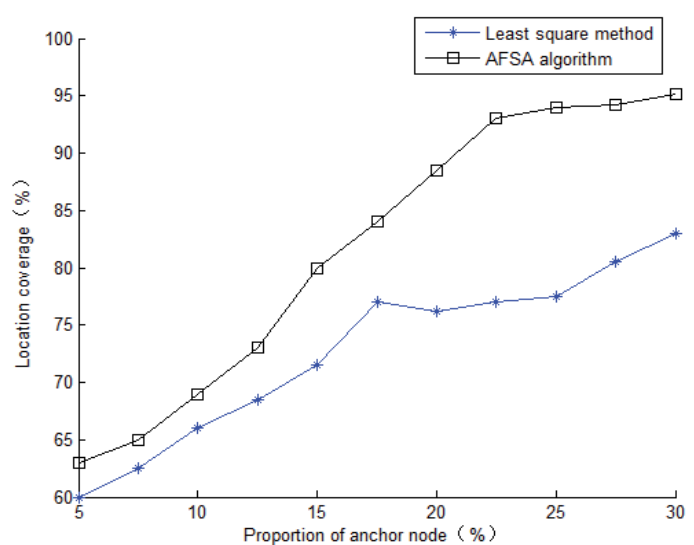

Figure 3. the influence of anchor node proportion on the location coverage

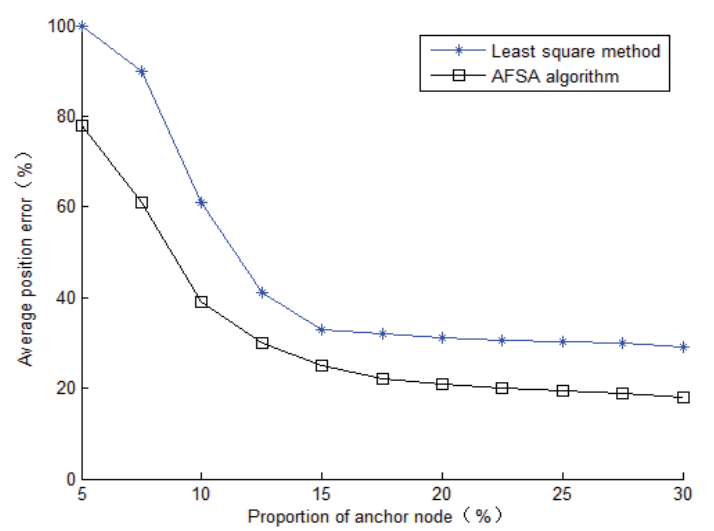

Figure 4. The influence of the ratio of beacon nodes on the average position error

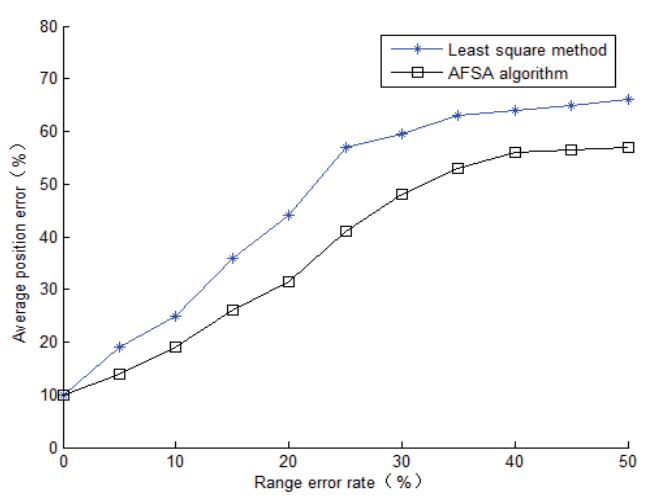

Figure 5. the influence of ranging error on the average position error

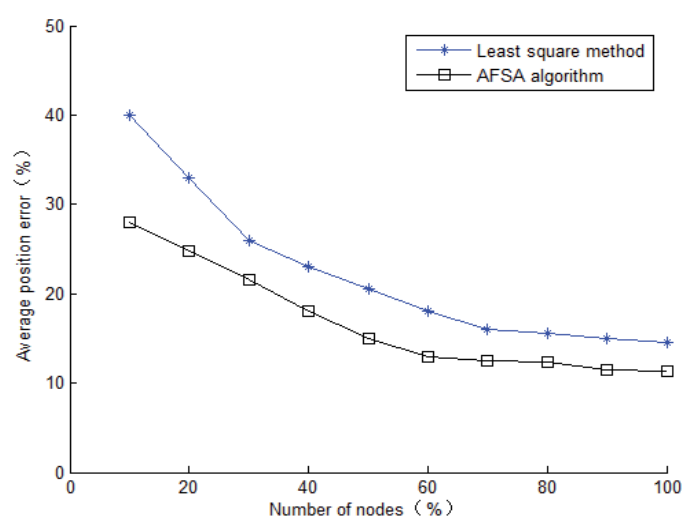

Figure 6. the relationship between the average position error and the number of nodes 
PAPER

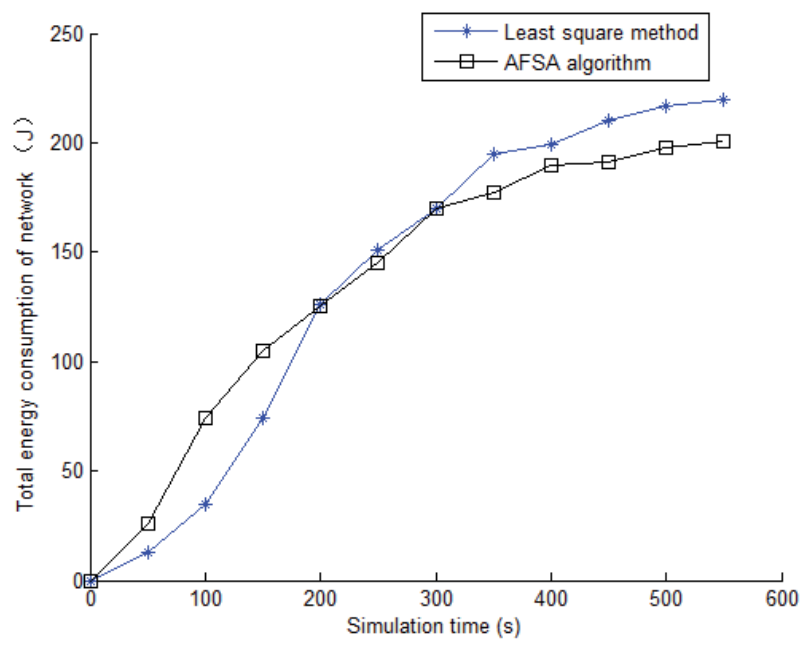

Figure 7. Comparison of the total energy consumption

less than the least square method. Because in the process of position, the unknown nodes can establish a nonlinear equation group with the distance information of the beacon nodes, and then estimate the coordinates of the unknown nodes by artificial fish swarm optimization algorithm. This shows that the communication cost of the algorithm is only the location information of the unknown node to the beacon node and the cost of computing nonlinear equations. Range method of AFSA algorithm and the least square method are the same, but computational quantity of the artificial fish swarm optimization algorithm is less than the least square method. So energy consumption of AFSA algorithm is less.

\section{CONCLUSIONS}

In order to improve the position accuracy of nodes in WSNs, a node localization algorithm based on artificial fish swarm algorithm is proposed in this paper. Firstly, location information of the beacon node and the TDOA technology is used to obtain the distance between the unknown node and the beacon node, and then calculate the coordinates of the unknown node by using artificial fish swarm algorithm. Because the algorithm has good global convergence, low initial value, strong robustness and other advantages, the algorithm only needs to compare the fitness of the objective function, and does not need the special information of the target function. So the algorithm can effectively reduce the influence of the error accumulation in the process of the positioning iteration. The simulation results show that the algorithm can improve the accuracy and the energy consumption is lower.

\section{REFERENCES}

[1] Sun, L., J. Li, Y. Chen, 2005. Wireless sensor networks. Beijing: Tsinghua University Press.

[2] Niculescu, D., B. Nath, "DV based positioning in ad hoc networks", Kluwer Journal of Telecommunication Systems, 22(1/4), 267-280,2003. http://dx.doi.org/10.1023/A:1023403323460
[3] Arivubrakan, P., V. R. S. Dhulipala,,'Energy consumption Heuristics in wireless sensor networks", Proceedings of the IEEE International Conference on Computing, Communication and Applications, Dindigul, Tamilnadu, 2012,pp.1-3.

[4] Ji, B. , L. Wang and Q. Yang, 2015. New Version of AES-ECC Encryption System Based on FPGA in WSNs, Journal of Software Engineering, 9 (1): 87-95. http://dx.doi.org/10.3923/jse.2015. 87.95

[5] Arivubrakan, P., V. R. S. Dhulipala, "Sentry Based Intruder Detection Technique for Wireless Sensor Networks", Journal of Artificial Intelligence, 2013,Vol. 6, pp. 175-180. http://dx.doi.org/10.3923/jai.2013.175.180

[6] Hu, X., M. Zhou and Q. Xia, "Internet of Things Positioning Model Study on Wireless Sensor Network", Information Technology Journal, 2013,Vol. 12 , pp. 8576-8582 http://dx.doi.org/10.3923/itj.2013.8576.8582

[7] Zhou, Y., A. Xin, S. Xia, "An improved APIT node selflocalization algorithm in WSN", Intelligent Control and Automation of 7th World Congress, Chongqing, 2008, pp. 7582-7586.

[8] Bulusu, N., J. Heidemann, D. Estrin, "GPS-less low-cost outdoor localization for very small devices", IEEE Personal Communications, 2000,Vol. 7, pp.28-34. http://dx.doi.org/10.1109/98.878533

[9] Savvides, A., C. Han, M. Strivastava, "Dynamic fine-grained localization in ad-hoc networks of sensors", The Annual International Conference on Mobile Computing and Networking. Rome, Italy,2001, pp.166-179. http://dx.doi.org/10.1145/381677.381693

[10] Niculescu, D., B. Nath, "Ad hoc positioning system (APS) using A0A", International Conference on Computer Communications. California, USA, 2003,pp. 1734-1743.

[11] Pi, W., "Wireless sensor network localization algorithm research", Jilin University, 2013

[12] Li, X., "A new intelligent optimization method for artificial fish swarm algorithm", Zhejiang: Zhejiang University, 2003.

[13] Xu, H., C. Bai, "Study on application of local neighborhood artificial fish swarm algorithm for $40 \mathrm{~Gb} / \mathrm{s}$ adaptive second-order polarization mode dispersion compensation", Optical and Quantum Electronics, 2012,Vol. 44, pp. 749-753. http://dx.doi.org/10.1007/s11082-012-9595-7

\section{AUTHORS}

Xiaoying Yang is with the College of Information Engineering, Suzhou University, Suzhou, China. (e-mail: yxiaoying2000@163.com).

Wanli Zhang is with College of Information Engineering, Suzhou University, Suzhou, China.

Qixiang Song is with the College of Information Engineering, Suzhou University, Suzhou, China.

The study of our work is supported in part by Young Talents Fund Project in Anhui Province of China (No.2012SQRL099ZD, No. 2013SQRL083ZD), and Anhui University Provincial Natural Science Research Project (No.KJ2014A247). Submitted 28 October 2015. Published as resubmitted by the authors 28 December 2015 . 